\title{
Fauna asociada a la palma de vino Attalea butyracea (Mutis ex L.f.) Wess.Boer (1988) (Arecales: Arecaceae) en un agroecosistema de Galeras, Sucre, Colombia
}

Fauna associated with the Wine Palm Attalea butyracea (Mutis ex L.f.) Wess.Boer (1988) (Arecales: Arecaceae) in an agroecosystem of Galeras, Sucre, Colombia

\section{Gerson A. Salcedo-Rivera, Fran Y. Herazo-Vitola, Juan F. Cruz y Oscar Sierra- Serrano}

\section{Resumen}

Se estudió la fauna asociada a Attalea butyracea en un agroecosistema de Galeras, Sucre, Colombia. Se efectuaron muestreos en junio de 2016 en la finca Mi Ranchito, donde se revisaron 42 palmas. De las palmas se extrajeron artrópodos y se buscaron vertebrados. Posteriormente, el material se identificó y se registraron 13 órdenes, con las siguientes frecuencias de aparición y abundancias: Hymenoptera (100 \%; 232), Coleoptera $(97,62 \% ; 240)$, Araneae $(95,24 \% ; 169)$, Blattodea $(88,09 \% ; 143)$, Hemiptera $(88,09 \%$; 82$)$, Amblypygi $(85,71 \%$; 166), Polydesmida $(78,57 \% ; 103)$, Lepidoptera $(73,81 \% ; 69)$, Archaeognatha $(71,43$ $\%$; 81), Orthoptera $(69,04 \%$; 31), Scorpiones $(69,04 \% ; 30)$, Ixodida $(66,66 \% ; 34)$ y Opiliones $(66,66 \% ; 57)$. Se identificaron las siguientes 18 especies: Arachnida: Charinus sp., Hapalopus sp., Phrynus barbadensis y Tityus tayrona; Insecta: Phileurus sp., Rhodnius pallescens y Triatoma dimidiata; Amphibia: Elachistocleis pearsei y Rhinella humboldti; Sauropsida: Bothrops asper, Cnemidophorus lemniscatus, Gonatodes albogularis, Gymnophthalmus speciosus y Mabuya sp.; y Mammalia: Artibeus lituratus, Didelphis marsupialis, Molossus sp. y Mus musculus. En conclusión, la palma de vino, por su arquitectura, funciona como microambiente para artrópodos y vertebrados pequeños; es utilizada como vivienda, dormitorio o refugio ocasional, lo que hace de esta planta un sitio importante en las interacciones de animales silvestres.

Palabras clave. Artrópodos. Ecosistema agrícola. Palma de vino. Vertebrados.

\begin{abstract}
The fauna associated with Attalea butyracea was studied in an agroecosystem of Galeras, Sucre, Colombia. Samplings were carried out during June 2016 in the farm Mi Ranchito, where 42 palms were checked. From the palms, arthropods were extracted and vertebrates were searched for. Subsequently, the material was identified and 13 orders were recorded with appearance frequencies and abundances as follows: Hymenoptera $(100 \%$; 232), Coleoptera $(97.62 \%$; 240), Araneae $(95.24 \%$; 169), Blattodea $(88.09 \%$; 143), Hemiptera $(88.09 \%$; 82), Amblypygi $(85.71 \%$; 166), Polydesmida $(78.57 \%$; 103), Lepidoptera $(73.81 \%$; 69), Archaeognatha $(71.43 \% ; 81)$, Orthoptera $(69.04 \%$; 31), Scorpiones $(69.04 \% ; 30)$, Ixodida $(66.66 \% ; 34)$ and Opiliones $(66.66 \%$; 57). Eighteen species were identified as follows: Arachnida: Charinus sp., Hapalopus sp., Phrynus barbadensis and Tityus tayrona; Insecta: Phileurus sp., Rhodnius pallescens and Triatoma dimidiata; Amphibia: Elachistocleis pearsei and Rhinella humboldti; Sauropsida: Bothrops asper, Cnemidophorus
\end{abstract}


lemniscatus, Gonatodes albogularis, Gymnophthalmus speciosus and Mabuya sp.; and Mammalia: Artibeus lituratus, Didelphis marsupialis, Molossus sp. and Mus musculus. In conclusion, the Wine Palm functions as a microenvironment for arthropods and small vertebrates due to its architecture; it is used as a dwelling, dormitory or occasional refuge, making it an important site for interactions of wild animals.

Keywords. Arthropods. Agricultural ecosystem. Wine palm. Vertebrates.

\section{Introducción}

Attalea butyracea, comúnmente conocida como la palma de vino o palma real, es una especie perteneciente a la familia Arecaceae (Arecales); se caracteriza por ser una palma de amplio grosor, solitaria, sin espinas, con raíces profundas, y puede alcanzar hasta $20 \mathrm{~m}$ de altura (CocomáReyes, 2010). Posee de 25 a 40 hojas grandes, pinnadas y ligeramente erectas, con las que se forma una corona densa (Devia et al., 2002). Es una especie monoica, con inflorescencias interfoliares, de las cuales las masculinas pueden medir entre 30 a $50 \mathrm{~cm}$ de longitud, mientras que las femeninas pueden alcanzar los 2,5 m de largo (CocomáReyes, 2010). Presenta abundantes flores de color blanco-amarillo, y sus frutos son pequeños, con medidas entre 4 y $5 \mathrm{~cm}$ (Cocomá-Reyes, 2010). Los frutos adquieren una coloración amarillenta en la madurez y contienen dos semillas oblongas (Pintaud, 2008).

La palma de vino es una especie vegetal masiva y abundante que se encuentra desde Centroamérica hasta el norte de América del Sur (Cocomá-Reyes, 2010), con un rango de distribución bajo los $300 \mathrm{~m}$ s.n.m., aunque ocasionalmente puede encontrarse hasta los 1000 m s.n.m. (Chang y Ling, 1998). En Colombia se distribuye en los valles de los ríos Cauca y Magdalena, la región Caribe, los Llanos Orientales y en la Amazonia (Henderson et al., 1995; Cocomá-Reyes, 2010). Se encuentra en bosques secos y estacionales, donde las poblaciones llegan a ser de miles de individuos (Cocomá-Reyes, 2010), así como en zonas perturbadas, pastizales, bordes de ríos y áreas abiertas como las sabanas (Cocomá-Reyes, 2010; Devia et al., 2002).

Esta arecácea posee una gran variedad de usos, siendo considerada como una de las especies vegetales de mayor utilidad en Colombia según Cocomá-Reyes (2010), quien menciona 36 usos, incluyendo forrajera, oleaginosa, medicinal, ornamental, artesanal y comestible; también constituye una fuente alimenticia importante para animales domésticos y silvestres (Chang y Ling, 1998).

En varias localidades del departamento de Sucre (costa norte de Colombia) se encuentran grandes extensiones de vegetación natural que han sido transformadas en potreros; como comentan Devia et al. (2002), en estos paisajes predominan varias especies de palmas que dan cierta estabilidad al ambiente, entre ellas $A$. butyracea, que además es una especie vegetal a la que se le atribuye un alto valor socioeconómico en el departamento. A partir de sus diferentes estructuras vegetales (hojas, tallo y frutos), se pueden obtener provechos de tipo gastronómico, artesanal y arquitectónico, que benefician a las poblaciones sucreñas (Devia et al., 2002). Además, su arquitectura, sobre todo los pecíolos persistentes en el tallo y la frondosa corona de hojas, forma una serie de microambientes que albergan a una gran cantidad de artrópodos y vertebrados pequeños, por lo que también se le reconoce una importancia ecológica (Chang y Ling, 1998); no obstante, la información sobre su papel como microhábitat de especies de fauna silvestre no se ha documentado formalmente en áreas sucreñas.

En esta investigación se pretendió contribuir al conocimiento sobre la artropofauna asociada a $A$. butyracea en una finca de Galeras, Sucre, Colombia, complementando con los registros de algunas especies de vertebrados encontrados en las palmas. 


\section{Materiales y métodos}

Área de estudio. El municipio de Galeras se encuentra localizado hacia el sector oriental del departamento de Sucre (Colombia) y hace parte de la subregión Sabanas. Presenta una altitud media de $70 \mathrm{~m}$ s.n.m., y limita con los municipios de Sincé (norte), San Benito Abad (sur y oeste), El Roble (oeste) y Magangué (departamento de Bolívar; este). Posee una extensión aproximada de $297 \mathrm{~km}^{2}$, compartiéndose entre el casco urbano y la zona rural, en la que se encuentra la finca Mi Ranchito $\left(09^{\circ} 08^{\prime} 22,6^{\prime \prime} \mathrm{N}-75^{\circ} 00^{\prime} 52,0^{\prime \prime} \mathrm{O} ; 80\right.$ $\mathrm{m}$ s.n.m.). Esta finca está dedicada a la ganadería bovina y a la agricultura, presentándose cultivos de yuca (Manihot esculenta), maíz (Zea mays) y ají (Capsicum апnuиm); es común encontrar árboles dispersos por la zona, como guásimos (Guazuma ulmifolia), junto con cercas de matarratón (Gliricidia sepium) que bordean el área, y también existe una vasta zona con una gran cantidad de palmas de vino dispersas, que comprenden una formación vegetal natural no cultivada (Figura 1).
Muestreo. En tres ocasiones durante junio de 2016 se llevaron a cabo visitas a la finca Mi Ranchito, con el fin de ubicar palmas de vino dentro del área, delas cuales se examinaron 42 en 3 muestreos diurnos con una duración de 6 horas por muestreo, en horario consecutivo desde las 08:00 h hasta las 14:00 $\mathrm{h}$.

En cada individuo se efectuó una revisión vertical, iniciando con una búsqueda activa y colecta manual de artrópodos desde el área del tallo que ajustaba con la superficie del suelo, pasando por los pecíolos persistentes en el tallo, hasta llegar a la frondosa corona de hojas, por lo que se necesitó el uso de una escalera. La revisión no solo incluyó la colecta de individuos que podían observarse externamente sobre la palma; también fue necesario escudriñar dentro del detrito acumulado en ciertas áreas como los peciolos y la corona. Todos los artrópodos extraídos fueron preservados en alcohol etílico al $70 \%$ dentro de recipientes plásticos independientes por palma.

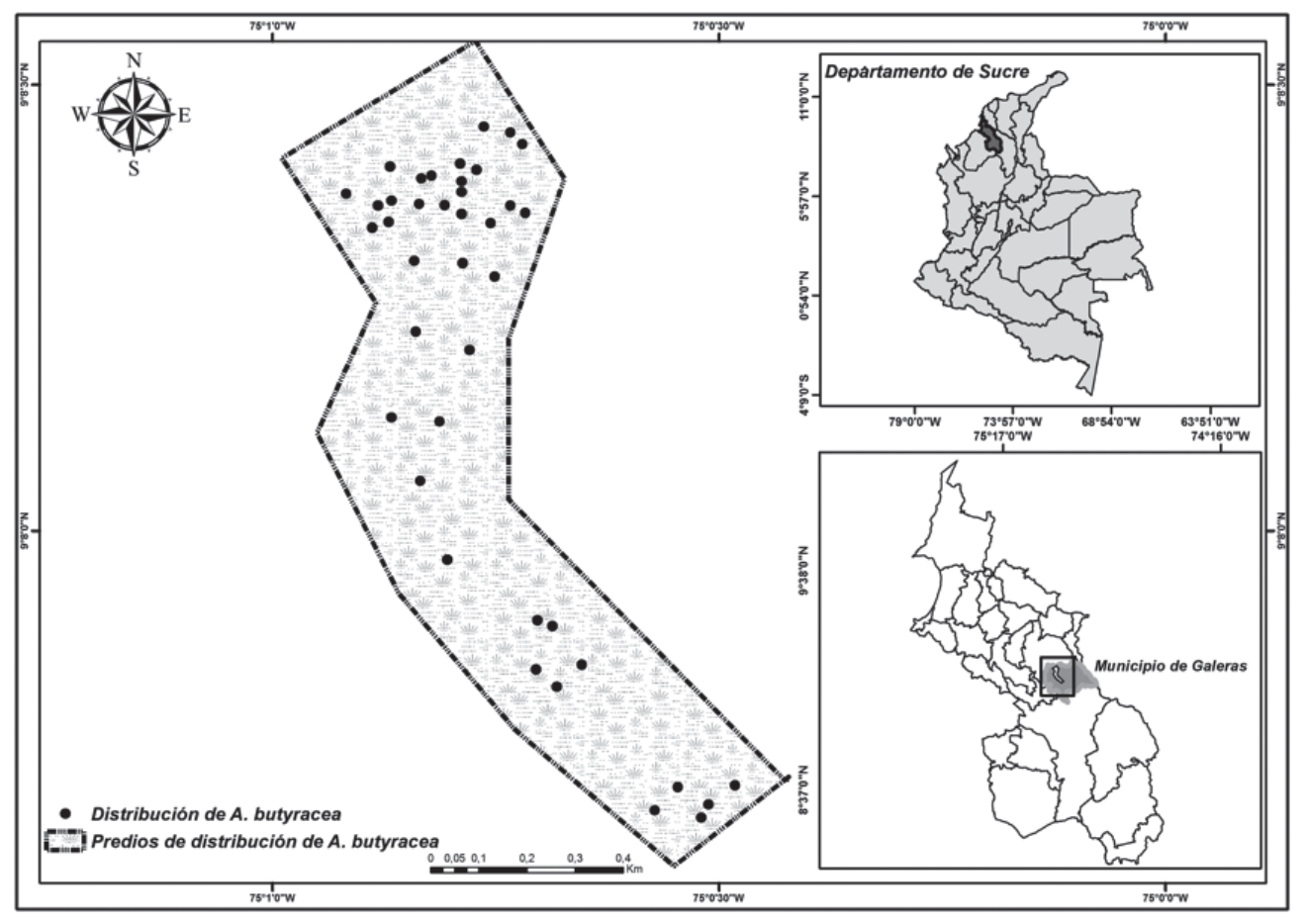

Figura 1. Área donde se realizó el estudio de la fauna asociada a Attalea butyracea (Arecaceae) en la finca Mi Ranchito, Galeras, Sucre, Colombia. 
Para complementar, se realizó una búsqueda activa de vertebrados en las distintas áreas de $A$. butyracea, tal y como se efectuó para los artrópodos, aunque también se revisó debajo de las hojas en descomposición de las palmas encontradas en el suelo próximo; sin embargo, los individuos solo fueron fotografiados y posteriormente liberados.

Fase de laboratorio. El material producto de la investigación se trasladó al Laboratorio de Conservación Biológica de la Universidad de Sucre (Sincelejo, Colombia) para su limpieza y conservación en alcohol etílico al $70 \%$, ubicando todo en recipientes plásticos independientes por cada una de las palmas para su posterior identificación taxonómica hasta nivel de orden. Se buscó una homogeneidad en identificación, y se emplearon las claves disponibles. Además, con la ayuda de expertos e investigadores, se logró la identificación de algunos ejemplares de artrópodos y vertebrados con las fotografías. Parte de la artropofauna se depositó por el momento en el Laboratorio de Fauna Silvestre de la Universidad de Sucre y otros ejemplares reposan en el Museo Zoológico de la Universidad de Sucre (MZUSU).

Análisis de datos. Se determinó la riqueza de órdenes como el número total deórdenes presentes en el estudio. La abundancia absoluta se calculó como el número total de individuos de un orden, mientras que la abundancia relativa se estableció como la relación, expresada porcentualmente, de individuos de un orden dado entre el número total de individuos colectados. Finalmente, se calculó la frecuencia de aparición para órdenes de artrópodos, dada por $\mathrm{Fr}=(\mathrm{pm} \times 100) / \mathrm{PM}$, donde pm era el número de palmas de muestreo en que se consignó el orden y PM era el número total de palmas muestreadas $(n=42)$.

\section{Resultados}

Se colectaron 1437 individuos distribuidos en 13 órdenes de artrópodos (Tabla 1), que representaron la composición de la artropofauna asociada a $A$. butyracea durante el tiempo de muestreo de esta investigación.
El orden con la mayor abundancia absoluta fue Coleoptera, que estuvo representado por 240 individuos $(16,70 \%)$, seguido de Hymenoptera con 232 (16,14 \%) (Tabla 1). Los grupos menos representativos en abundancia de individuos fueron Orthoptera $(31 ; 2,16 \%)$ y Scorpiones (30; 2,09\%) (Tabla 1).

Hymenoptera fue el orden de artrópodos que se registró en todas las palmas muestreadas, teniendo, por tanto, una frecuencia de aparición del 100 \% (Figura 2); Coleoptera también estuvo bien representado en cuanto a su presencia en las palmas, con una frecuencia de aparición de 97,62 $\%$ (41 palmas). Orthoptera con 69,04 \% (29 palmas) junto con Acari $(66,66 \%$; 28 palmas) fueron los órdenes con las frecuencias de aparición más bajas (Figura 2).

Tabla 1. Abundancias absolutas y relativas para los órdenes de artrópodos asociados a Attalea butyracea.

\begin{tabular}{ccc}
\hline Orden & $\begin{array}{c}\text { Abundancia } \\
\text { absoluta }\end{array}$ & $\begin{array}{c}\text { Abundancia } \\
\text { relativa } \\
\mathbf{( \% )}\end{array}$ \\
\hline Coleoptera & 240 & 16,70 \\
\hline Hymenoptera & 232 & 16,14 \\
\hline Araneae & 169 & 11,76 \\
\hline Amblypygi & 166 & 11,55 \\
\hline Blattodea & 143 & 9,95 \\
\hline Polydesmida & 103 & 7,17 \\
\hline Hemiptera & 82 & 5,71 \\
\hline Archaeognatha & 81 & 5,64 \\
\hline Lepidoptera & 69 & 4,80 \\
\hline Opiliones & 57 & 3,97 \\
\hline Ixodida & 34 & 2,37 \\
\hline Orthoptera & 31 & 2,16 \\
\hline Scorpiones & 30 & 2,09 \\
\hline Total & 1437 & \\
\hline
\end{tabular}


De igual forma, se lograron identificar 18 especies entre invertebrados y vertebrados que se encontraron en las palmas durante la búsqueda (Tabla 2).

Los anfibios fueron detectados dentro de algunas oquedades formadas entre la superficie del suelo y la base de las palmas, y ocultos entre las hojas en descomposición localizadas en la superficie del suelo cercana a las mismas.
Para el caso de los reptiles, las especies registradas fueron localizadas en el interior de los peciolos y la corona de hojas de las palmas, así como los mamíferos (Figura 3).

Se destaca que los triatominos (Hemiptera: Reduviidae: Triatominae) se colectaron solo en las palmas donde se encontraron mamíferos.

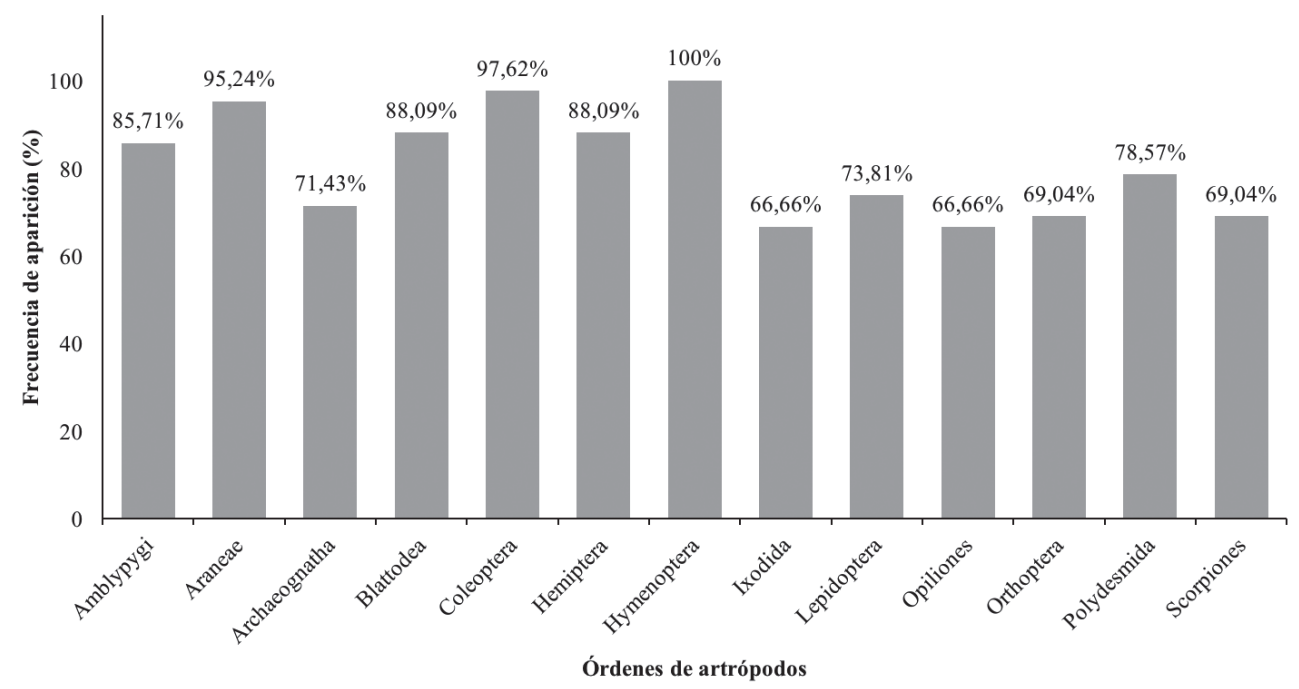

Figura 2. Diagrama de barras para las frecuencias de aparición de los órdenes de artrópodos encontrados en las palmas de estudio.

Tabla 2. Invertebrados y vertebrados encontrados en Attalea butyracea.

\begin{tabular}{|c|c|c|c|}
\hline Clase & Orden & Familia & Especie \\
\hline \multirow{4}{*}{ Arachnida } & \multirow{2}{*}{ Amblypygi } & Charinidae & Charinus sp. \\
\hline & & Phrynidae & Phrynus barbadensis (Pocock, 1893) \\
\hline & Araneae & Theraphosidae & Hapalopus sp. \\
\hline & Scorpiones & Buthidae & Tityus tayrona Lourenço, 1991 \\
\hline \multirow{3}{*}{ Insecta } & Coleoptera & Melolonthidae & Phileurus sp. \\
\hline & \multirow{2}{*}{ Hemiptera } & \multirow{2}{*}{ Reduviidae } & Rhodnius pallescens Barber, 1932 \\
\hline & & & Triatoma dimidiata (Latreille, 1811) \\
\hline \multirow{2}{*}{ Amphibia } & \multirow{2}{*}{ Anura } & Bufonidae & Rhinella humboldti (Gallardo, 1965) \\
\hline & & Microhylidae & Elachistocleis pearsei (Ruthven, 1914) \\
\hline
\end{tabular}


Cont. Tabla 2. Invertebrados y vertebrados encontrados en Attalea butyracea.

\begin{tabular}{|c|c|c|c|}
\hline Clase & Orden & Familia & Especie \\
\hline \multirow{5}{*}{ Sauropsida } & \multirow{5}{*}{ Squamata } & Gymnophthalmidae & $\begin{array}{l}\text { Gymnophthalmus speciosus (Hallowell, } \\
\text { 1861) }\end{array}$ \\
\hline & & Scincidae & Mabuya sp. \\
\hline & & Sphaerodactylidae & $\begin{array}{l}\text { Gonatodes albogularis (Duméril \& Bibron, } \\
1836 \text { ) }\end{array}$ \\
\hline & & Teiidae & $\begin{array}{l}\text { Cnemidophorus lemniscatus (Linnaeus, } \\
1758 \text { ) }\end{array}$ \\
\hline & & Viperidae & Bothrops asper (Garman, 1884) \\
\hline \multirow{4}{*}{ Mammalia } & \multirow{2}{*}{ Chiroptera } & Molossidae & Molossus sp. \\
\hline & & Phyllostomidae & Artibeus lituratus Olfers, 1818 \\
\hline & Didelphimorphia & Didelphidae & Didelphis marsupialis Linnaeus, 1758 \\
\hline & Rodentia & Muridae & Mus musculus Linnaeus, 1758 \\
\hline
\end{tabular}
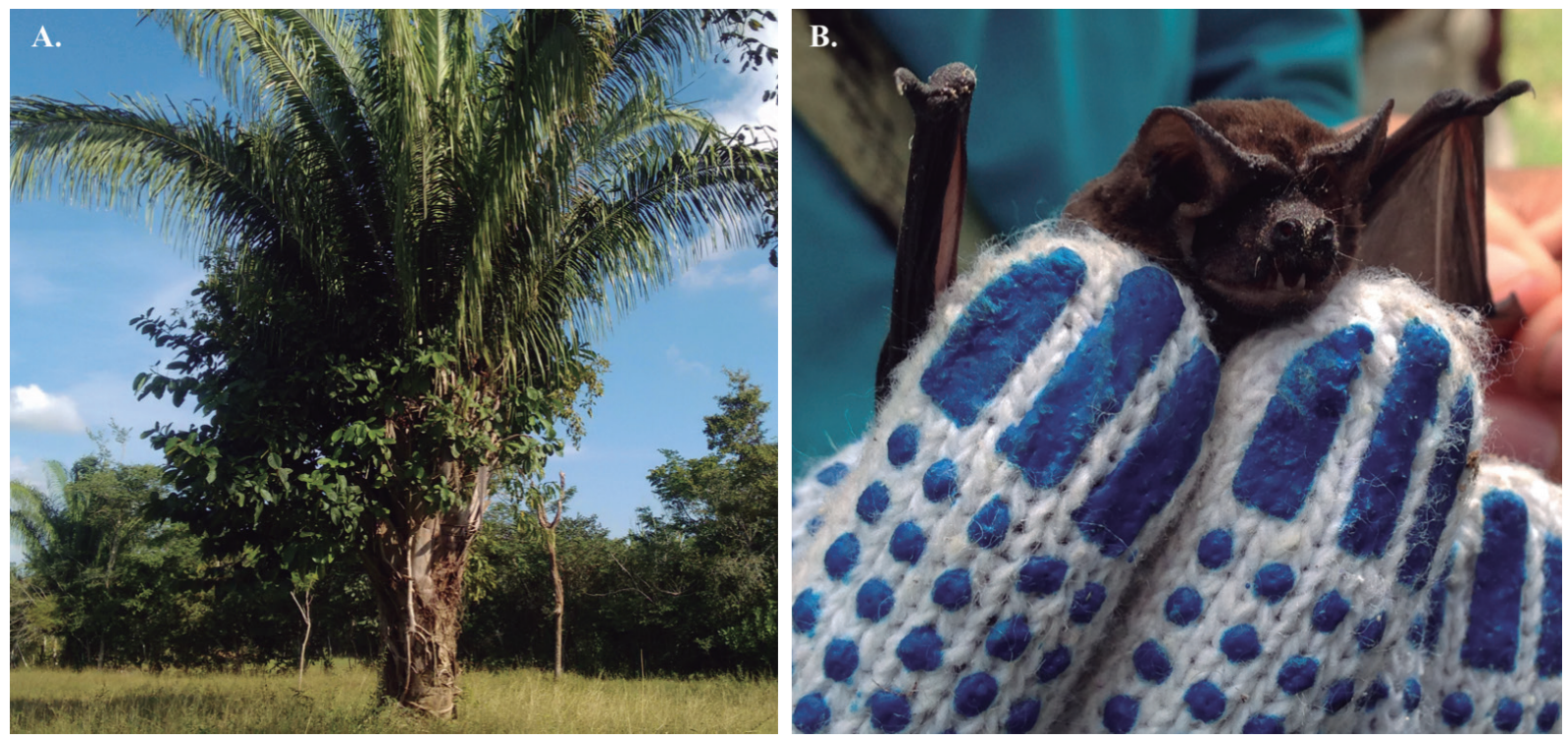

Figura 3. A) Palma de vino (Attalea butyracea) en la finca Mi Ranchito (Galeras, Sucre, Colombia). B) Molossus sp. encontrado en la corona de hojas de Attalea butyracea. 


\section{Discusión}

Chang y Ling (1998) afirman que la palma de vino es una planta que se considera como la única especie arbórea presente en matorrales y potreros. Su presencia en estas áreas es de gran importancia para la fauna local, ya que los peciolos persistentes en el tallo y la frondosa corona de hojas son microambientes para comunidades de artrópodos y vertebrados pequeños, los cuales la utilizan para diversos fines, tales como vivienda, alimentación, lugar de percha y/o caza, o refugio ocasional. Animales domésticos y silvestres usan la gran producción de flores y frutos como una fuente alimenticia importante en sus dietas, en especial en zonas alteradas en las que fuentes de alimento natural están restringidas o no se encuentran disponibles (Chang y Ling, 1998).

Los resultados de esta investigación concuerdan con los registrados en la literatura sobre artropofauna asociada a especies de palmas. Chang y Ling (1998) presentaron una lista de fauna asociada con $A$. butyracea en seis comunidades del Área de Conservación AmistadPacífico (Costa Rica), en la que registraron, similar a esta investigación, grupos como escorpiones, amblipígidos, opiliones, arañas, colémbolos, hemípteros, himenópteros, coleópteros, ortópteros, blatodeos y lepidópteros. Asimismo, Gaona-García et al. (2014), en su trabajo sobre la interacción planta-animal de la palma camedora (Chamaedorea radicalis) en la Reserva de la Biósfera "El Cielo" (México), encontraron insectos, entre los que destacaron Orthoptera, Hemiptera, Lepidoptera, Hymenoptera y Coleoptera.

No es de extrañar que órdenes como Coleoptera e Hymenoptera presentaran la mayor abundancia en los muestreos, porque se podría decir que esto es un reflejo de la propia diversidad de dichos grupos, que ocupan los primeros lugares entre los insectos (Pérez-Solache et al., 2015; Montero et al., 2011). En trabajos como el de Lara-Villalón et al. (2015) se destacó que la presencia de Coleoptera e Hymenoptera en especies de palmas puede estar relacionada con que algunas especies de estos órdenes tienden a causar daños forestales en las plantaciones, mientras que otras especies de estos grupos no representan un problema fitosanitario para cultivos de palmas.

La presencia de arácnidos como Phrynus barbadensis y Charinus sp. en palmas está bien documentada en la literatura; Chang y Ling (1998) identificaron que los amblipígidos explotan los recursos de la arquitectura de la palma de vino para así utilizarla como vivienda. Sobre Hapalopus sp. no se halló información de su asociación con palmas, aunque en el estudio de Chang y Ling (1998) se encontraron arañas en las palmas de vino, pero los autores no definieron el uso que estas le daban a la palma. Acerca de la ocurrencia de Tityus tayrona en las palmas de vino, Salazar y Calle (2003) afirman que estas sirven de refugio y alimento para los escorpiones.

Algunos estudios han confirmado la ocurrencia de ciertos escarabajos en especies de palmas, los cuales tienen incidencia negativa sobre estas, siendo defoliadores y dañando el follaje mediante perforaciones, defoliación de bordes y puntas, y raspado del haz (Gaona-García et al., 2014; García-Atencia y Martínez-Hernández, 2015); sin embargo, Chang y Ling (1998) afirman que algunas especies de escarabajos también utilizan a A. butyracea como vivienda.

La fauna de triatominos ha sido ampliamente estudiada debido a su importancia epidemiológica (Salazar y Calle, 2003; Guhl et al., 2007); en Colombia varias investigaciones han tratado el tema de la caracterización ecoepidemiológica de algunas especies de triatominos asociados a palmas (Salazar y Calle, 2003; Guhl et al., 2007; Parra-Henao et al., 2016). Los resultados presentes, con respecto a triatominos en la palma de vino, se relacionan con las publicaciones acerca del tema; las dos especies encontradas en esta investigación también han sido encon-tradas en A. butyracea y otras palmas en dife-rentes regiones del país. Con este trabajo se complementa la información acerca de los eco-topos naturales de triatominos, como lo son las palmas. Esto tiene gran significado en el sentido epidemiológico, puesto que Mesa et al. (1991) presentaron 
un caso de miocarditis chagásica aguda en un niño de dos años de edad en el área rural del municipio de Galeras; la pesquisa epidemiológica en la zona permitió identificar que el vector peridomiciliario era Rhodnius pallescens, habitante normal de la palma de vino según los investigadores, y del que se aisló Trypanosoma cruzi.

Con respecto a la herpetofauna, MorazánFernández et al. (2013) presentaron un trabajo en el que se estudió la relación entre la fauna silvestre y las plantaciones de palma africana (Elaeis guineensis) en Costa Rica; encontraron un total de 1915 individuos registrados, de los cuales 814 eran anfibios y reptiles. Estos autores también definen que, para la mayoría de las especies de anfibios y reptiles, las plantaciones de palma sirven como zonas de refugio y sitios de reproducción, lo que concuerda con parte de lo comentado por Chang y Ling (1998), quienes afirman que algunas especies de la herpetofauna utilizan la palma de vino como vivienda, aunque otras especies, como algunas serpientes, pueden usarla como dormitorio o para la caza. Es de enfatizar que, con respecto a estos dos estudios, la única especie que se comparte con los resultados de esta investigación es Gonatodes albogularis; sin embargo, los anfibios encontrados ya han sido estudiados en áreas con presencia de E. guineensis (Blanco-Torres et al., 2015; Yepes et al., 2016), donde se ha reconocido a Rhinella humboldti en asociación a senderos secos dentro del cultivo y borde del cultivo en general (Yepes et al., 2016).

Acerca de los mamíferos, Morazán-Fernández et al. (2013) capturaron 18 especies de murciélagos, de las cuales, en relación a los resultados presentes, solo Artibeus lituratus es compartida, y con respecto a didélfidos (Didelphimorphia: Didelphidae), encontraron cinco especies, compartiéndose a Didelphis marsupialis; además, Chang y Ling (1998) presentaron 15 especies de mamíferos asociados a la palma de vino. No obstante, en ninguno de los dos trabajos anteriores se presentaron ratones como parte de la mastofauna encontrada en las palmas. Según Chang y Ling (1998), algunas especies, como los filostómidos
(Chiroptera: Phyllostomidae) y D. marsupialis, tienden a aprovechar a las palmas para efectos de alimentación y vivienda; sin embargo, otros mamíferos, especialmente murciélagos, la usan también para caza o percha (Hernández-Mijangos y Medellín, 2013; Rodríguez-Herrera et al., 2007; García-García y Santos-Moreno, 2014). Por último, la presencia de mamíferos en las palmas en las que también se colectaron los triatominos debe ser considerado como de interés epidemiológico; según Reyes et al. (2017), el mantenimiento de ecotopos, que pueden alojar animales como triatominos y mamíferos (entre ellos zarigüeyas, murciélagos y ratones), permite también mantener el ciclo silvestre de la enfermedad de Chagas.

Finalmente, se destaca que, en relación a la composición de vertebrados asociados, las diferencias entre trabajos anteriores con A. butyracea y otras palmas con respecto al presente, pueden asociarse con que no se utilizó una metodología estricta para la captura.

\section{Conclusiones}

Características morfológicas que definen la arquitectura de $A$. butyracea, como los peciolos persistentes en el tallo y la frondosa corona de hojas, permiten el establecimiento de un microambiente en el que se produce la estancia e interacción entre invertebrados y vertebrados pequeños. Resulta necesario continuar con estudios faunísticos a profundidad y con métodos específicos de captura para grupos animales asociados a $A$. butyracea y otras palmas, incluyendo, además, investigaciones con la flora asociada a estas, y anexando la evaluación de las características microclimáticas (temperatura, humedad, entre otras) que propician que alberguen tal diversidad biológica, para así considerarlas como elemento importante para la conservación.

\section{Agradecimientos}

Los autores agradecen a la familia Mejía Martínez por su hospitalidad durante la estancia en la finca Mi Ranchito. A Rubén Camargo, Dairo Carrascal, 
José Cobo y Holver Narváez por su colaboración y asistencia durante la fase de campo de la investigación. A Tatiana Vergara y Wendy Zabala por su apoyo en las actividades de laboratorio. A Pedro Atencia, Carolina Ayala, Andrés Blanco, Eduar Borja, Ricardo Borja, Cristian Castillo, Carlos Hernández, doctor Jhon Neita, Luis Romero y Angie Tinoco por su ayuda en la identificación y corroboración de algunas especies. A los doctores Jaime De La Ossa, Alcides Sampedro y Liliana Solano, y demás revisores anónimos del manuscrito, por sus importantes aportes para la redacción final.

\section{Referencias}

Blanco-Torres, A., Duré, M. y Bonilla, M. A. (2015). Observaciones sobre la dieta de Elachistocleis pearsei y Elachistocleis panamensis en dos áreas intervenidas de tierras bajas del norte de Colombia. Revista Mexicana de Biodiversidad, 86, 538-540.

Chang, Y. y Ling, F. (1998). Caracterización biológica y de uso de Palma Real (Attalea butyracea Mutis ex L.f.) en 6 comunidades del área de conservación Amistad Pacífico, Costa Rica. Costa Rica: Instituto Nacional de Biodiversidad de Costa Rica. 25 pp.

Cocomá-Reyes, C. (2010). Uso y manejo de la palma real (Attalea Butyracea) para la elaboración de artesanías en el departamento del Tolima (Trabajo de grado). Colombia: Pontificia Universidad Javeriana, Facultad de Ciencias, departamento de Biología. 40 pp.

Devia, J., López, A. y Saldarriaga, O. L. (2002). Productos promisorios del fruto de palma de vino. Revista Universidad EAFIT, 126, 67-80.

Gaona-García, G., Lara-Villalón, M., TrejoHernández, L. y Sánchez-Ramos, G. (2014). Interacción planta-animal de la palma camedora en la Reserva de la Biósfera "El Cielo", Tamaulipas, México. Entomología Mexicana, 1, 547-551.

García-Atencia, S. y Martínez-Hernández, N. (2015). Escarabajos fitófagos (Coleoptera:
Scarabaeidae) del departamento del Atlántico, Colombia. Acta Zoológica Mexicana (n.s.), 31(1), 89-96.

García-García, J. L. y Santos-Moreno, A. (2014). Use of Attalea butyracea palm as tent-roosting by Uroderma bilobatum bat in Oaxaca, Mexico. Acta Zoológica Mexicana (n.s.), 30(2), 434-438.

Guhl, F., Aguilera, G., Pinto, N. y Vergara, D. (2007). Actualización de la distribución geográfica y ecoepidemiología de la fauna de triatominos (Reduviidae: Triatominae) en Colombia. Biomédica, 27 (Supl. 1), 143-162.

Henderson, A., Galeano, G. y Bernal, R. (1995). Field guide to the palms of the Americas. Estados Unidos: Princeton University Press. 352 pp.

Hernández-Mijangos, L. A. y Medellín, R. A. (2013). Use of Tents by the Big Fruit-Eating Bat Artibeus lituratus (Chiroptera: Phyllostomidae) in Chiapas, Mexico. The Southwestern Naturalist, 58, 107-109.

Lara-Villalón, M., Rosas-Mejía, M., Rojas-Fernández, P. y Reyes-Castillo, P. (2015). Hormigas (Hymenoptera: Formicidae) asociadas a palma camedora (Chamaedorea radicalis Mart.) en el bosque tropical, Gómez Farías, Tamaulipas, México. Acta Zoológica Mexicana (n.s.), 31(2), 270-274.

Montero, G., Carnevale, N. y Magra, G. (2011). Ensambles estacionales de artrópodos epigeos en un bosque de quebracho (Schinopsis balansae) en el Chaco Húmedo. Revista Colombiana de Entomología, 37(2), 294-304.

Morazán-Fernández, F., Gutiérrez Sanabria, D. R., Coello-Toro, H. L., Arévalo-Huezo, E., Ioli, A. G., Díaz Gutiérrez, N., Guerra, L. F., Burbano, D., Guevara, C., Lobos, L., Rico-Urones, A., CortésSuárez, J. E., Jiménez, R., Reinke, H., Narváez, V. y Aranda, J. M. (2013). Relación entre la fauna silvestre y las plantaciones de palma africana (Elaeis guineensis) y su efecto en la producción de pequeños y medianos productores en la Península deOsa, Costa Rica. Costa Rica: Instituto Internacional de Conservación y Manejo de Vida Silvestre, Universidad Nacional de Costa Rica. 104 pp. 
Parra-Henao, G., Quirós-Gómez, O., Jaramillo, N. y Segura Cardona, A. (2016). Environmental determinants of the distribution of Chagas disease vector Triatoma dimidiata in Colombia. Am. J. Trop. Med. Hyg., 94(4), 767-774.

Pérez-Solache, A., Herrerías-Diego, Y., PonceSaavedra, J. (2015). Variación en las comunidades de artrópodos epigeos en sitios de bosque de pino en proceso de restauración. Biológicas, 17(2), 43-52.

Pintaud, J. C. (2008). An orverview of the taxonomy of Attalea (Arecaceae). Perú Biológica, 15, 55-63.

Reyes, M., Torres, A., Esteban, L., Flórez, M. y Angulo, V. M. (2017). Riesgo de transmisión de la enfermedad de Chagas por intrusión de triatominos y mamíferos silvestres en
Bucaramanga, Santander, Colombia. Biomédica, 37, 68-78.

Rodríguez-Herrera, B., Medellín, R. A. y Timm, R. M. (2007). Murciélagos neotropicales que acampan en hojas / Neotropical tent-roosting bats. Costa Rica: Editorial Inbio. 184 pp.

Salazar, D. y Calle, J. (2003). Caracterización ecoepidemiológica de Rhodnius pallescens en la palma Attalea butyracea en la región momposina (Colombia). Actual. Biol., 25(78), 31-38.

Yepes, Y., Acuña-Vargas, J. C. y Bastidas, B. (2016). Análisis trófico del sapito común Rhinella humboldti (Gallardo, 1965) asociada a sistemas intervenidos de bosque seco en zona rural del municipio de Dibulla -La Guajira. Ciencia e Ingeniería, 3(1), 8-15.
Gerson A. Salcedo-Rivera

Universidad de Sucre,

G.I. en Biodiversidad Tropical

Sincelejo, Colombia

gsalcedo07@hotmail.com

Fran Y. Herazo-Vitola

Universidad de Sucre,

G.I. Evolución y Sistemática Tropical

Sincelejo, Colombia

foitola19@yahoo.com

Juan F. Cruz

Universidad de Sucre,

G.I. Evolución y Sistemática Tropical

Sincelejo, Colombia

juanfcruzm@gmail.com

\section{Oscar Sierra-Serrano}

Universidad de Sucre,

Facultad de Educación y Ciencias

Sincelejo, Colombia

oscarsaw09@gmail.com
Fauna asociada a la palma de vino Attalea

butyracea (Mutis ex L.f.) Wess.Boer (1988)

(Arecales: Arecaceae) en un agroecosistema de Galeras, Sucre, Colombia

Citación del artículo: Salcedo-Rivera, G. A., Herazo-Vitola., F. Y., Cruz, J. F. y Sierra-Serrano, O. (2018). Fauna asociada a la palma de vino Attalea butyracea (Mutis ex L.f.) Wess.Boer (1988) (Arecales: Arecaceae) en un agroecosistema de Galeras, Sucre, Colombia. Biota Colombiana, 19(1), 39-48. DOI: 10.21068/c2018.v19n01a03.

Recibido: 20 de enero de 2018

Aprobado: 20 de abril de 2018 